\title{
Reliability updating with measurements in spatially distributed systems using stochastic simulation
}

\author{
D. Straub \\ Engineering Risk Analysis Group, TU München, Germany
}

\begin{abstract}
A recently developed method for reliability updating with equality information [Straub D., Probabilistic Engineering Mechanics, under review] is investigated for application to spatially distributed systems. In contrast to existing ones, the novel method enables the use of stochastic simulation (e.g. Monte Carlo or importance sampling) for reliability updating with any type of information. The main benefit of this approach over existing methods is that it is robust and calculations can thus be automated. This is of particular relevance for spatially distributed systems subject to uncertain information (e.g. though measurements, monitoring, observations of system performances), which require a large number of conditional reliability calculations. In the current paper it is shown that for such systems, stochastic simulation in combination with the novel method can provide accurate results for a large number of locations simultaneously.
\end{abstract}

\section{INTRODUCTION}

We are concerned with computing the probability of a rare event $F$ (the failure event) conditional on uncertain information, e.g. as obtained from measurements, inspection or monitoring of structures. Such Bayesian updating has been applied in the context of structural reliability since the 1970s (e.g. Tang 1973). The conditional probability of failure given the information event $Z$ is computed as

$$
\operatorname{Pr}(F \mid Z)=\frac{\operatorname{Pr}(F \cap Z)}{\operatorname{Pr}(Z)}
$$

In structural reliability, failure events $F$ and information events $Z$ are described by domains $\Omega$ in the outcome space of the basic random variables $\mathbf{X}=\left(X_{1}, X_{2}, \ldots, X_{n}\right)$. The failure domain $\Omega_{F}$ is defined in terms of continuous limit state functions $g(\mathbf{x})$. In the simplest case, it is

$$
\Omega_{F}=\{g(\mathbf{x}) \leq 0\}
$$

In the general case, $\Omega_{F}$ is defined in terms of a number of limit state functions (e.g., Der Kiureghian 2005), corresponding to systems of components that are defined by limit state functions. For the purpose of the present paper, the formulation in (2) is sufficiently general; extension to the system application is straightforward (Straub 2010).

Information obtained on the system, e.g. in the form of measurements, monitoring, inspection, observed system performance, is also described in terms of continuous limit state functions $h(\mathbf{x})$ and corresponding domains. Information is said to be of the inequality type if it can be written as

$$
\Omega_{Z}=\{h(\mathbf{x}) \leq 0\}
$$


and it is said to be of the equality type if it can be written as

$$
\Omega_{Z}=\{h(\mathbf{x})=0\}
$$

Structural reliability methods (SRM) solve Eq. (1) by computing integrals in the space of $\mathbf{X}$ :

$$
\operatorname{Pr}(F \mid Z)=\frac{\int_{\mathbf{x} \in\left\{\Omega_{F} \cap \Omega_{Z}\right\}} f(\mathbf{x}) \mathrm{d} \mathbf{x}}{\int_{\mathbf{x} \in\left\{\Omega_{Z}\right\}} f(\mathbf{x}) \mathrm{d} \mathbf{x}}
$$

If information is exclusively of the inequality type, Eq. (3), evaluation of the above integrals is straightforward using any of the available SRM. However, if the information event $Z$ is of the equality type, the integrals result in zero, since these events have zero probability a-priori. Direct application of SRM is thus not possible in this case.

Solutions to overcome this problem have been suggested by Madsen (1987) and the group of Rackwitz (e.g. Schall et al. 1988). Madsen's solution is based on inserting a dummy random variable and then computing probability sensitivities with respect to this variable. The solutions of the Rackwitz group are based on computing surface integrals, using first- or second order approximations of the surfaces $h_{i}(\mathbf{x})=0$. These solutions are implemented in the Strurel software (Gollwitzer et al. 2006). Both Madsen's and Rackwitz' methods are efficient and, in many cases, represent a sufficiently accurate approximation. However, in cases where FORM/SORM solutions are not sufficiently accurate or in which it is difficult to identify the joint design point, these methods should not or cannot be used. Furthermore, it is often difficult to appraise the error made by the first- or second-order approximation.

Recently, the author has proposed a novel method for solving Eq. (5) using SRM when information is of the equality type (Straub 2010). The method is based on transforming equality information into inequality information, which enables the direct use of Eq. (5) using any SRM. The aim of the present paper is to study the application of the methodology to reliability problems involving spatially distributed systems. For such systems, commonly a large number of reliability problems must be solved simultaneously, which requires that the applied algorithms are robust and efficient. Here, it is investigated if and how stochastic simulation approaches are suitable in this context, whereby particular focus is put on examining the trade-off between computational efficiency (measured in number of evaluations of the limit state functions) and robustness (i.e. the applicability of a method without the need for problem specific adjustments). First- and second order reliability methods (FORM/SORM), which are highly efficient but not necessarily robust and accurate in all cases, are not investigated here. They are considered in Straub (2010). Additionally, Papaioannou and Straub (2010) utilize subset simulation in conjunction with the methodology for reliability updating in stochastic FEM.

\section{BAYESIAN UPDATING WITH EQUALITY INFORMATION}

This section presents a summary of the method described in Straub (2010), with slight modifications in view of the application of stochastic simulation methods.

\subsection{Describing information through likelihood functions}

We note that in statistics, information is not commonly described in the form of domains $\Omega_{Z}$. Instead, the usual way to describe (uncertain) information on $\mathbf{X}$ is by means of the likelihood function, which is defined as

$$
L(\mathbf{x}) \propto \operatorname{Pr}(Z \mid \mathbf{X}=\mathbf{x})
$$

As noted in Straub (2010), any domain $\Omega_{Z}$ can be translated into a likelihood function. However, it is often more convenient to directly identify the likelihood function. As an example, consider a measurement $s_{m}$ of a system characteristic $s(\mathbf{X})$. The measurement has an additive error $\varepsilon$ that is a zero mean random variable uncorrelated with $\mathbf{X}$. The limit state function $h(\mathbf{X}, \varepsilon)$ describing this equality information as well as the corresponding likelihood function are given in the following, with $f_{\varepsilon}()$ being the PDF of $\varepsilon$. 


$$
\begin{aligned}
& h(\mathbf{X}, \varepsilon)=s(\mathbf{X})-s_{m}+\varepsilon \\
& L(\mathbf{x})=f_{\varepsilon}\left(s_{m}-s(\mathbf{x})\right)
\end{aligned}
$$

For the case of several observations $Z_{1}, \ldots, Z_{m}$ with corresponding likelihood functions $L_{i}(\mathbf{x})$, it is always possible to combine the likelihood functions into a single likelihood function $L(\mathbf{x})$. E.g., if measurements are uncorrelated for given $\mathbf{X}=\mathbf{x}$, it is simply $L(\mathbf{x})=\prod_{i=1}^{m} L_{i}(\mathbf{x})$. It is thus sufficient to consider only the case of a single likelihood function describing the combined observations $Z=Z_{1} \cap \ldots \cap Z_{m}$ in the following.

\subsection{Transform equality information into equivalent inequality information}

Let $P$ be a random variable with uniform distribution in the range $[0,1]$ and let $c$ be a constant that is selected so that $0 \leq c L(\mathbf{x}) \leq 1$ for all $\mathbf{x}$. In this case, the following identity holds for given values of $\mathbf{X}=\mathbf{x}$ :

$$
L(\mathbf{x})=\frac{\operatorname{Pr}[P \leq c L(\mathbf{x})]}{c}
$$

Let $\alpha$ denote the proportionality constant in the likelihood definition given in Eq. (6). By combining with Eq. (9), we obtain

$$
\operatorname{Pr}(Z \mid \mathbf{X}=\mathbf{x})=\alpha L(\mathbf{x})=\frac{\alpha}{c} \operatorname{Pr}[P \leq c L(\mathbf{x})]
$$

It follows that the probability of the information event $Z$ is

$$
\operatorname{Pr}(Z)=\int_{\mathbf{X}} \operatorname{Pr}(Z \mid \mathbf{X}=\mathbf{x}) f(\mathbf{x}) \mathrm{d} \mathbf{x}=\frac{\alpha}{c} \int_{\mathbf{X}}[P \leq c L(\mathbf{x})] f(\mathbf{x}) \mathrm{d} \mathbf{x}
$$

Next, we define the event $Z_{e}=\{P \leq c L(\mathbf{x})\}$ through the limit state function

$$
h_{e}(\mathbf{x}, p)=p-c L(\mathbf{x})
$$

and the corresponding domain $\Omega_{z e}=\left\{h_{e}(\mathbf{x}, p) \leq 0\right\}$. This has the same form as the domains describing inequality information, Eq. (3). Equation (11) can now be rewritten to

$$
\operatorname{Pr}(Z)=\frac{\alpha}{c} \int_{\mathbf{x}, p \in \Omega_{Z e}} f(p) f(\mathbf{x}) \mathrm{d} \mathbf{x} \mathrm{d} p=\frac{\alpha}{c} \int_{\mathbf{x}, p \in \Omega_{Z e}} f(\mathbf{x}) \mathrm{d} \mathbf{x} \mathrm{d} p
$$

The second identity follows from $f(p)=1$. Accordingly, $\operatorname{Pr}(F \cap Z)$ is obtained as

$$
\operatorname{Pr}(F \cap Z)=\int_{\mathbf{X}} \operatorname{Pr}(Z \mid \mathbf{X}=\mathbf{x}) \operatorname{Pr}(F \mid \mathbf{X}=\mathbf{x}) f(\mathbf{x}) \mathrm{d} \mathbf{x}=\frac{\alpha}{C} \int_{\mathbf{x}, p \in\left\{\Omega_{F} \cap \Omega_{Z \ell}\right\}} f(\mathbf{x}) \mathrm{d} \mathbf{x} \mathrm{d} p
$$

The conditional probability of $F$ given $Z$ is therefore

$$
\operatorname{Pr}(F \mid Z)=\frac{\int_{\mathbf{x}, p \in\left\{\Omega_{F} \cap \Omega_{Z e}\right\}} f(\mathbf{x}) \mathrm{d} \mathbf{x} \mathrm{d} p}{\int_{\mathbf{x}, p \in \Omega_{Z e}} f(\mathbf{x}) \mathrm{d} \mathbf{x} \mathrm{d} p}
$$

Here, the proportionality constant $\alpha$ disappears. Both integrals in Eq. (15) can be computed using any SRM. The denominator corresponds to a component reliability problem, the nominator to a parallel system reliability problem.

As shown in Straub (2010), the limit state surfaces $h_{e}(\mathbf{x}, p)=0$, which describe the information, are generally highly non-linear. For this reason, there it was recommended not to apply FORM/SORM for the evaluation, unless the shape of the limit state surface is well understood. This is not generally the case for problems involving more than a few random variables and few observations. This motivates the use of stochastic simulation methods for the evaluation of the integrals in Eq. (15). 


\section{STOCHASTIC SIMULATION FOR COMPUTING THE CONDITIONAL PROBABILITY}

Based on the transformation of equality information into inequality information, basic stochastic simulation methods (Monte Carlo, importance sampling) are adapted to the computation of the conditional probability $\operatorname{Pr}(F \mid Z)$ in this section. Other methods are available and further improvements of the suggested methods are possible and are under investigation.

\subsection{Monte Carlo evaluation}

The use of Monte Carlo Simulation (MCS) for the evaluation of the integrals in (15) has two main advantages: It is easy to implement and it provides unbiased results, which do not depend on choices made by the analyst. For these reasons, it is suggested to use MCS whenever possible for the verification of the results obtained with other methods.

The disadvantage of MCS is the required large number of evaluations of the limit state functions $g(\mathbf{x})$ and $h_{e}(\mathbf{x}, p)$ when the events $F$ and $Z_{e}$ have small probabilities of occurrence. In structural reliability, $\operatorname{Pr}(F \mid Z)$ is commonly required to be small and MCS is therefore not applicable for problems where the evaluation of $g(\mathbf{x})$ is computationally expensive. Typically these are problems involving finite element models (FEM), e.g. Papaioannou and Straub (2010). In addition, even for problems that have a parametrical function $g(\mathbf{x})$, MCS might not be feasible because of small values of $\operatorname{Pr}\left(Z_{e}\right)$. Nonetheless, there are a number of applications for which MCS represents a viable solution strategy. An example of MCS applied to reliability updating of a structural component subject to fatigue crack growth is presented in Straub (2010).

With MCS, the integrals in Eq. (15) are approximated by

$$
\operatorname{Pr}(F \mid Z) \approx \frac{\sum_{i=1}^{n_{s}} I\left[h_{e}\left(\mathbf{x}_{i}, p_{i}\right) \leq 0\right] I\left[g\left(\mathbf{x}_{i}\right) \leq 0\right]}{\sum_{i=1}^{n_{s}} I\left[h_{e}\left(\mathbf{x}_{i}, p_{i}\right) \leq 0\right]}=\frac{n_{F Z}}{n_{Z}}
$$

where $n_{s}$ is the number of samples, $\mathbf{x}_{i}$ is the $i$ th sample of $\mathbf{X}, p_{i}$ is the $i$ th sample of $P$ and $I[$ ] is the indicator function that takes value 1 if the condition holds and 0 otherwise. The denominator in Eq. (16), $n_{Z}$, is the number of sample that fall into the domain $\Omega_{Z e}$; it can be interpreted as the effective number of samples that are available to compute the probability of $F$ conditional on $Z$. Its expected value is $\mathrm{E}\left[N_{Z}\right]=n \operatorname{Pr}\left(Z_{e}\right)$. If $\operatorname{Pr}\left(Z_{e}\right)>>\operatorname{Pr}\left(F \cap Z_{e}\right)$, we can neglect the variance of $N_{Z}$ and the standard deviation of the MCS estimator is approximately

$$
\sigma_{M C S} \approx \sqrt{\frac{\operatorname{Pr}\left(F \cap Z_{e}\right)-\operatorname{Pr}\left(F \cap Z_{e}\right)^{2}}{n \operatorname{Pr}\left(Z_{e}\right)}}
$$

As the accuracy of the MCS estimator is a function of $\operatorname{Pr}\left(Z_{e}\right)=\operatorname{Pr}\left(h_{e}(\mathbf{x}, p) \leq 0\right)$, its applicability depends on the available information $Z$. As will illustrated later in the numerical example, $\operatorname{Pr}\left(Z_{e}\right)$ decreases with increasing number of observations, but is also a function of the dependence among the observations. Generally, the higher the information content of the observations with respect to the basic random variables $\mathbf{X}$, the lower $\operatorname{Pr}\left(Z_{e}\right)$ will become.

\subsubsection{Selecting the constant $c$}

$\operatorname{Pr}\left(Z_{e}\right)$ is directly proportional to the chosen value of the constant $c$ in Eq. (9). To increase the efficiency of MCS, it is thus beneficial to select the largest possible value of $c$ that still fulfills the condition $0 \leq c L(\mathbf{x}) \leq 1$.

If the location of the maximum value of $L(\mathbf{x})$ in the space of $\mathbf{X}$ is known (this is the Maximum Likelihood Estimator $\left.\mathbf{x}_{M L E}\right)$, the constant can be selected as $c=1 / L\left(\mathbf{x}_{M L E}\right)$. When applying MCS, a simpler solution is to select $c$ by computing the likelihood for all samples of $\mathbf{X}$ and setting it equal to the inverse of the largest observed value:

$$
c=\frac{1}{\max \left[L\left(\mathbf{x}_{i}\right)\right]}
$$


In doing so, the value of the likelihood function is truncated at $1 / c$, as evident from Eq. (9). This has no influence on the result obtained with MCS, however, because the likelihood function $L(\mathbf{x})$ is not evaluated in the regions of $\mathbf{X}$ where $L(\mathbf{x})$ is truncated.

\subsection{Importance sampling}

MCS becomes inefficient for problems with more than a few random variables and a few observations. Importance sampling (IS) can provide a more efficient estimation of $\operatorname{Pr}(F \mid Z)$. General introductions to IS in reliability applications are given e.g. in (Engelund and Rackwitz 1993).

The IS estimator for the conditional probability $\operatorname{Pr}(F \mid Z)$ in Eq. (15) is

$$
\operatorname{Pr}(F \mid Z) \approx \frac{\sum_{i=1}^{n_{s}} I\left[h_{e}\left(\mathbf{x}_{i}, p_{i}\right) \leq 0\right] I\left[g\left(\mathbf{x}_{i}\right) \leq 0\right] \frac{f_{\mathbf{X}}\left(\mathbf{x}_{i}\right)}{\psi\left(\mathbf{x}_{i}, p_{i}\right)}}{\sum_{i=1}^{n_{s}} I\left[h_{e}\left(\mathbf{x}_{i}, p_{i}\right) \leq 0\right] \frac{f_{\mathbf{X}}\left(\mathbf{x}_{i}\right)}{\psi\left(\mathbf{x}_{i}, p_{i}\right)}}
$$

wherein the samples $\mathbf{x}_{i}$ and $p_{i}$ are simulated from a distribution with sampling density $\psi(\mathbf{x}, p)$. The efficiency of importance sampling is determined by the selected sampling density $\psi(\mathbf{x}, p)$. It is not possible to generically define an optimal sampling density that is valid for all applications; however, it is possible to split $\psi(\mathbf{x}, p)$ into

$$
\psi(\mathbf{x}, p)=\psi_{1}(\mathbf{x}) \psi_{2}(p \mid \mathbf{x})
$$

where $\psi_{1}(\mathbf{x})$ is the sampling PDF of $\mathbf{X}$ and $\psi_{2}(p \mid \mathbf{x})$ is the conditional sampling density of $P$ given $\mathbf{X}=\mathbf{x}$. An optimal conditional sampling density $\psi_{2}(p \mid \mathbf{x})$ that is valid for any application of Eq. (19) is given as

$$
\psi_{2}(p \mid \mathbf{x})=\frac{1}{c L(\mathbf{x})}, \quad 0 \leq p \leq c L(\mathbf{x})
$$

If it holds that $L(\mathbf{x})>0$ for any $\mathbf{x}$, then $I\left[h_{e}\left(\mathbf{x}_{i}, p_{i}\right) \leq 0\right]=1$ for any value of $p_{i}$ that is sampled from the above conditional density $\psi_{2}(p \mid \mathbf{x})$. In this case, Eq. (19) reduces to

$$
\operatorname{Pr}(F \mid Z) \approx \frac{\sum_{i=1}^{n_{s}} I\left[g\left(\mathbf{x}_{i}\right) \leq 0\right] \frac{f_{\mathbf{X}}\left(\mathbf{x}_{i}\right) L\left(\mathbf{x}_{i}\right)}{\psi_{1}\left(\mathbf{x}_{i}\right)}}{\sum_{i=1}^{n_{s}} \frac{f_{\mathbf{X}}\left(\mathbf{x}_{i}\right) L\left(\mathbf{x}_{i}\right)}{\psi_{1}\left(\mathbf{x}_{i}\right)}}
$$

Note that the constant $c$ disappears when using this sampling density.

The optimal choice of $\psi_{1}(\mathbf{x})$ is problem dependent. Ideally, $\psi_{1}(\mathbf{x})$ should be centered around the area of interest in the space of $\mathbf{X}$. If it is possible to identify the design point of the event $Z_{e}$ and the joint design point of the event $\left\{Z_{e} \cap F\right\}$, the sampling densities $\psi_{1}(\mathbf{x})$ can be centered around these points. This approach was successfully applied in (Straub 2010), and for many problems such a strategy works well. For the identification of the design points, efficient algorithms exist and are implemented in commercial software (e.g., Strurel, Gollwitzer et al. 2006). However, for spatially distributed systems requiring the computation of the reliability probability at many locations, the approach might not be sufficiently robust in all cases, since the identification of the design points requires solving many optimization problems simultaneously.

As an alternative, a robust choice for $\psi_{1}(\mathbf{x})$ is the a-priori PDF of $\mathbf{X}, f_{\mathbf{X}}(\mathbf{x})$. If the prior probability of failure is relatively large (say $\beta<3$ ), this sampling density can provide reasonably accurate results with limited number of samples. With $\psi_{1}(\mathbf{x})=f_{\mathbf{X}}(\mathbf{x})$, the estimate of the conditional probability in Eq. (22) simplifies further to

$$
\operatorname{Pr}(F \mid Z) \approx \frac{\sum_{i=1}^{n_{s}} I\left[g\left(\mathbf{x}_{i}\right) \leq 0\right] L\left(\mathbf{x}_{i}\right)}{\sum_{i=1}^{n_{s}} L\left(\mathbf{x}_{i}\right)}
$$


It should be evident that this estimate can be interpreted as a Monte Carlo estimate wherein each sample is weighted with its corresponding likelihood. The same result could have be obtained by noting that the posterior PDF of $\mathbf{X}$ is $f_{\mathbf{x}}^{\prime \prime}(\mathbf{x} \mid Z)=\alpha L(\mathbf{x}) f(\mathbf{x})$, with constant

$$
a=\frac{1}{\int_{\mathbf{X}} L(\mathbf{x}) f(\mathbf{x}) \mathrm{d} \mathbf{x}}
$$

and that $\operatorname{Pr}(F \mid Z)$ can be calculated from $f_{\mathbf{X}}^{\prime \prime}(\mathbf{x} \mid Z)$ by

$$
\operatorname{Pr}(F \mid Z)=\int_{\mathbf{X}} I[g(\mathbf{x}) \leq 0] f^{\prime \prime}(\mathbf{x} \mid Z) \mathrm{d} \mathbf{x}=\frac{\int_{\mathbf{X}} I[g(\mathbf{x}) \leq 0] L(\mathbf{x}) f(\mathbf{x}) \mathrm{d} \mathbf{x}}{\int_{\mathbf{X}} L(\mathbf{x}) f(\mathbf{x}) \mathrm{d} \mathbf{x}}
$$

The solution in Eq. (23) is the MCS estimator of the two integrals in Eq. (25).

\section{EXAMPLE: OBSERVATIONS OF A GAUSSIAN PROCESS}

This example is included to investigate and illustrate the performance of the proposed method and the different computation approaches. Let $\mathbf{X}=\left[X_{1}, X_{2}, \ldots, X_{n}\right]^{\mathrm{T}}$ be the values of a Gaussian process at $n=100$ locations with coordinates $\mathbf{l}=[1,2, \ldots, 100]^{\mathrm{T}}$. All $X_{i}$ have zero mean and unit variance; the covariance structure is fully described by the autocovariance function

$$
\operatorname{Cov}\left(X_{i}, X_{j}\right)=\exp \left(-\frac{\left|l_{i}-l_{j}\right|}{20}\right)
$$

At each location $l_{i}$, the failure event $i$ is defined as the variable $X_{i}$ exceeding value 2, i.e.,

$$
g_{i}(\mathbf{x})=2-x_{i}
$$

We consider $n_{o}$ observations $y_{i}$ of the process at different locations $l_{i}, i=1, \ldots, n_{o}$. Each observation is associated with an uncorrelated Gaussian error, with zero mean and standard deviation 0.5 . The likelihood function is thus

$$
\mathrm{L}(\mathbf{x})=\prod_{i=1}^{n_{0}} \frac{1}{0.5 \sqrt{2 \pi}} \exp \left[-\frac{1}{2}\left(\frac{y_{i}-x_{l(i)}}{0.5}\right)^{2}\right]
$$

For the numerical analysis, we consider three scenarios, summarized in Table 1.

Table 1. Measurements considered for the reliability updating.

\begin{tabular}{lllll}
\hline \multicolumn{2}{l}{ Case $n_{o}$} & Locations $l_{i}$ & Observations $y_{i}$ & $\operatorname{Pr}\left(Z_{e}\right)$ \\
\hline a & 2 & 30,70 & $1,-1$ & 0.077 \\
b & 5 & $10,30,50,70,90$ & $0.5,0.5,0.5,0.5,0.5$ & 0.016 \\
c & 10 & $5,15,25, \ldots, 85,95$ & $0.0,0.5,0.5,0.0,-0.5,0.5,0.0,0.5,0.5,0.0$ & 0.0014 \\
\hline
\end{tabular}

In the last column of Table 1 , the probability of the equivalent information event $\operatorname{Pr}\left(Z_{e}\right)$ is provided. Here, $\operatorname{Pr}\left(Z_{e}\right)$ is calculated with MCS, whereby $Z_{e}$ is defined with $c=1 / L\left(\mathbf{x}_{M L E}\right)$.

\subsection{Monte Carlo Simulation}

The results obtained with MCS using $10^{5}$ samples are summarized in Figure 1, together with the true reliability calculated analytically. In case (a), MCS provides useful results with reasonably close credible intervals (credible intervals are the Bayesian equivalent of confidence intervals), with the exception of those areas that exhibit large posterior reliability. In case (b) MCS results are suitable for the purpose of verifying results obtained with importance sampling methods (or 
other methods, e.g. FORM/SORM), but are not sufficiently accurate for most other purposes. This is mainly due the fact that the probability of the equivalent information event $\operatorname{Pr}\left(Z_{e}\right)$ reduces with increased number of measurements, Table 1 , and therefore the effective number of samples $n_{Z}$ also diminishes according to Eq. (16). In case (c), due to the same reasons, no result can be obtained with MCS. Here, additionally, the increased number of measurements reduces the uncertainty and, therefore, increases the reliability, which leads to a reduction in the accuracy of MCS.
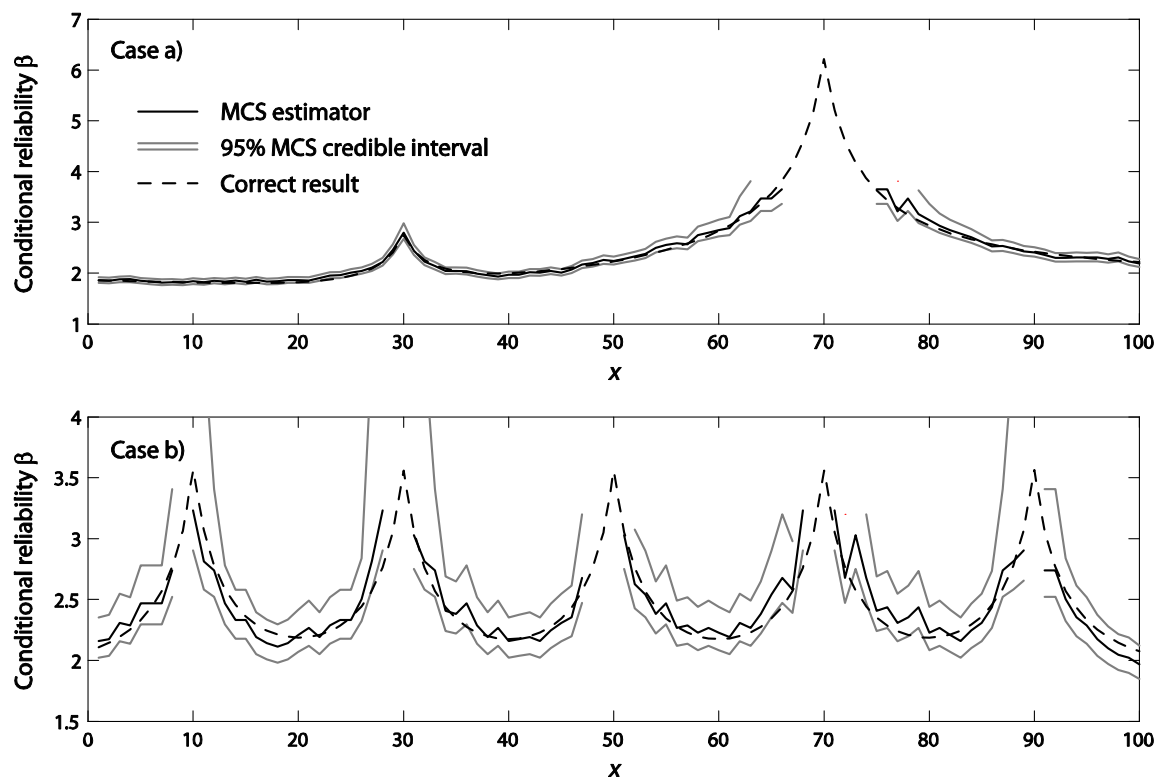

Figure 1. Results obtained with MCS with $10^{5}$ samples.

\subsection{Importance sampling}

We investigate importance sampling with the conditional sampling density $\psi_{2}(p \mid \mathbf{x})$ selected as the optimal one, Eq. (21), and with two different sampling densities $\psi_{1}(\mathbf{x})$, listed in the following.

1. The prior PDF of $\mathbf{X}, \psi_{1}(\mathbf{x})=f_{\mathbf{X}}(\mathbf{x})$;

2. Multinormal distribution with mean equal to the design point corresponding to failure of element $i$ and covariance matrix equal to that of $\mathbf{X}, C_{\mathbf{X X}}$.

With the first choice of $\psi_{1}(\mathbf{x})$, the sampling density is the same for the evaluation of the conditional reliability at all locations $l_{i}$. The second choice involves a different $\psi_{1}(\mathbf{x})$ for the evaluation of the conditional reliability at every location $l_{i}$. However, the design points applied here are readily obtained: It suffices to identify the design point $u_{i}$ of the variable $X_{i}$ (which is the same for every $l_{i}$ and in the present example is simply equal to $u_{i}=2$ ) and then determine the conditional mode of the other variables given $X_{i}=u_{i}$. Other investigated choices for $\psi_{1}(\mathbf{x})$ did not lead to significant improvement in accuracy over these two choices. As an example, the use of a sampling density $\psi_{1}(\mathbf{x})$ located around the MLE leads to similar accuracy than choice 1 above.

The results obtained with importance sampling are summarized in Figure 2 below. Not surprisingly, the choice of a different sampling density for the evaluation of the conditional reliability at every location $l_{i}$ (choice 1 ) leads to higher accuracy in all cases. However, this choice does require a higher computational effort than the use of a common $\psi_{1}(\mathbf{x})$ for all locations. As evident from Figure 2, the accuracy decreases with increasing number of measurements. This effect is similar to that observed in the application of MCS.

Computation of the results presented here takes in the order of 50CPU seconds on a standard PC with a $1.6 \mathrm{GHz}$ processor. The computation time is approximately linear to the number of simulations. 

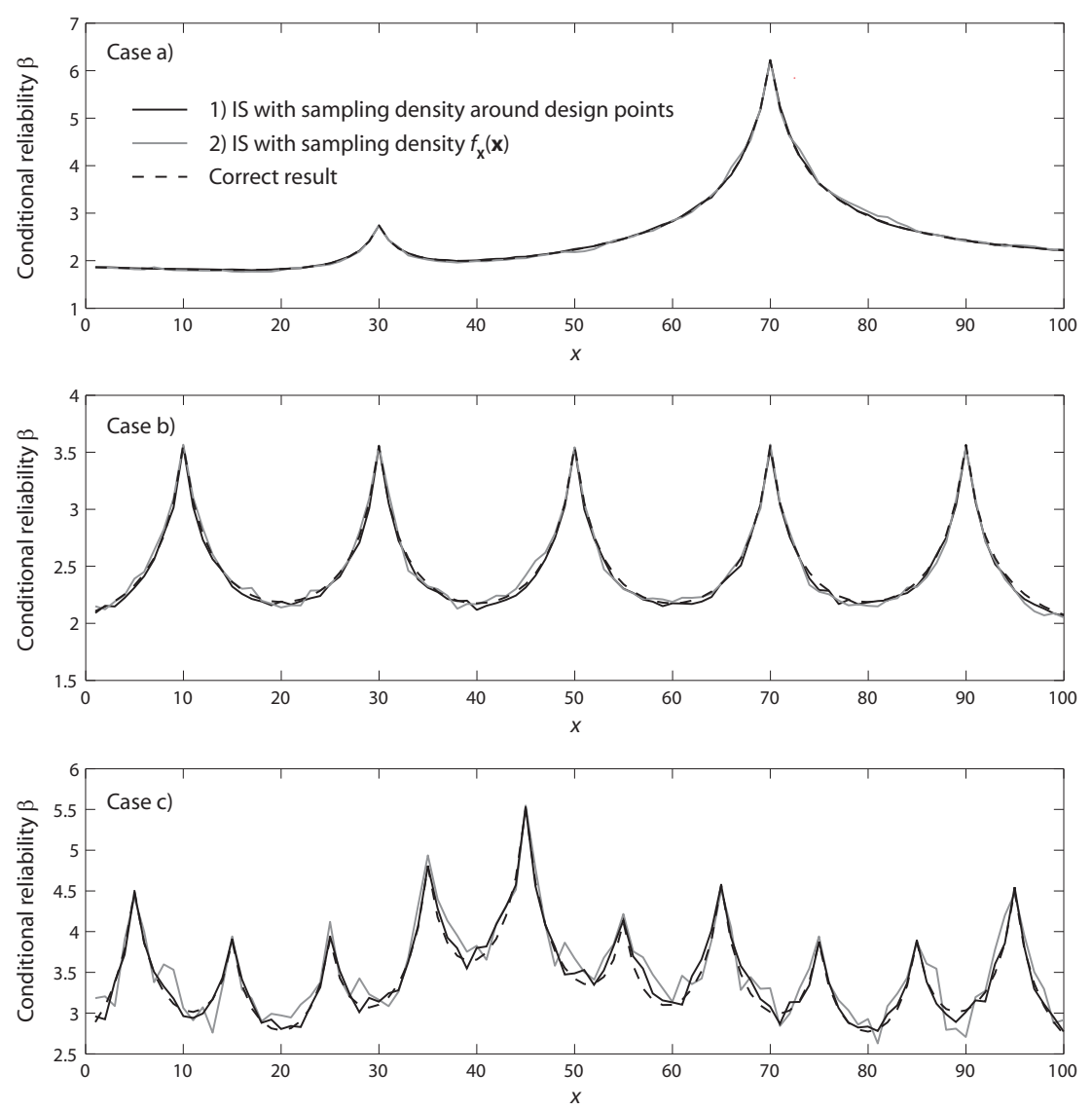

Figure 2. Results obtained with importance sampling with $10^{5}$ samples.

\section{CONCLUSIONS}

With the novel approach proposed by the author in (Straub 2010), which is summarized in this paper, Bayesian updating in the context of structural reliability can be performed with stochastic simulation. In the present paper, the use of Monte Carlo simulation and Importance Sampling techniques is investigated. The approach has strong potential for reliability updating in spatially distributed systems, where robust algorithms are required, due to the large number of conditional reliability evaluations. Potential applications are manifold, including reliability updating of deteriorating large-scale engineering systems following inspections and monitoring results.

\section{REFERENCES}

Der Kiureghian, A. (2005), First- and second-order reliability methods. Chapter 14, in Engineering design reliability handbook, edited by E. Nikolaidis et al., CRC Press, Boca Raton, FL.

Engelund, S., and R. Rackwitz (1993), A benchmark study on importance sampling techniques in structural reliability, Structural Safety, 12(4), 255-276.

Gollwitzer, S., B. Kirchgäßner, R. Fischer, and R. Rackwitz (2006), PERMAS-RA/STRUREL system of programs for probabilistic reliability analysis, Structural Safety, 28(1-2), 108-129.

Madsen, H. O. (1987), Model Updating in Reliability Theory, in Proc. ICASP 5, Vancouver, Canada.

Papaioannou, I. and D. Straub (2010). Geotechnical reliability updating using stochastic FEM. Proc. IFIP WG7.5 Working Conference on Reliability and Optimization of Structural Systems, Munich.

Schall, G., S. Gollwitzer, and R. Rackwitz (1988), Integration of multinormal densities on surfaces, in Proc. 2nd IFIP WG 7.5 Working Conference, London.

Straub, D. (2010), Reliability updating with equality information. Probabilistic Engineering Mechanics, under review.

Tang, W. H. (1973), Probabilistic Updating of Flaw Information, Journal of Testing and Evaluation, 1(6), 459-467. 